\title{
THE VLA LIGHT CURVES OF 0957+561, 1979-1994
}

\author{
D. B. HAARSMA, J. N. HEWITT AND B. F. BURKE \\ Massachusetts Institute of Technology \\ AND
}

J. LEHÁR

Harvard-Smithsonian Center for Astrophysics

\begin{abstract}
The gravitational lens $0957+561$ has been monitored by our group for 16 years, using the VLA at $5 \mathrm{GHz}$. Since our last report in 1992, both images have returned to their quiescent flux levels, and the A image has brightened again. We apply various analysis methods to the light curves, and obtain a preliminary estimate of $\tau=455 \pm 40$ days for the time delay.
\end{abstract}

Since its discovery (Walsh et al. 1979), we have monitored 0957+561 using the VLA at $5 \mathrm{GHz}$. There are currently 111 good observations up to December 1994, taken approximately every month. We map the VLA data and measure the fluxes of the two quasar images using the techniques of Lehár et al. (1992). The new light curves are presented in Figure 1. Since 1990, the fluxes of both the A and B images have decreased back to their quiescent levels, and the A image has risen again in flux. The strong fluctuation in the B image in the spring of 1990 appears to be anomalous, but these data calibrated without problems and produced good quality maps, so they are not bad data points. When the curves are lined up at delays greater than 500 days, it can be seen that the $\mathrm{A}$ image does not decrease fast enough in 1991 to match the B image flux, so long delays seem to be excluded by the new feature.

The method of Press et al. $(1992 a, 1992 b)$ uses a maximum likelihood estimator based on a structure function analysis of the light curves. Press et al. found a $\chi^{2}$ minimum to the 1979-1990 data of $\tau=548_{-16}^{+10}$ days (95\% C.L.) and flux ratio $R=0.694 \pm 0.002$; there is also a secondary minimum around $\tau=455$ days and $R=0.698$. Using the same analysis on the 1979-1994 data, we find that the $\chi^{2}$ minimum has shifted to $\tau=455$ days and $R=0.698$ (see Figure 2). Note that there is still a local minimum 


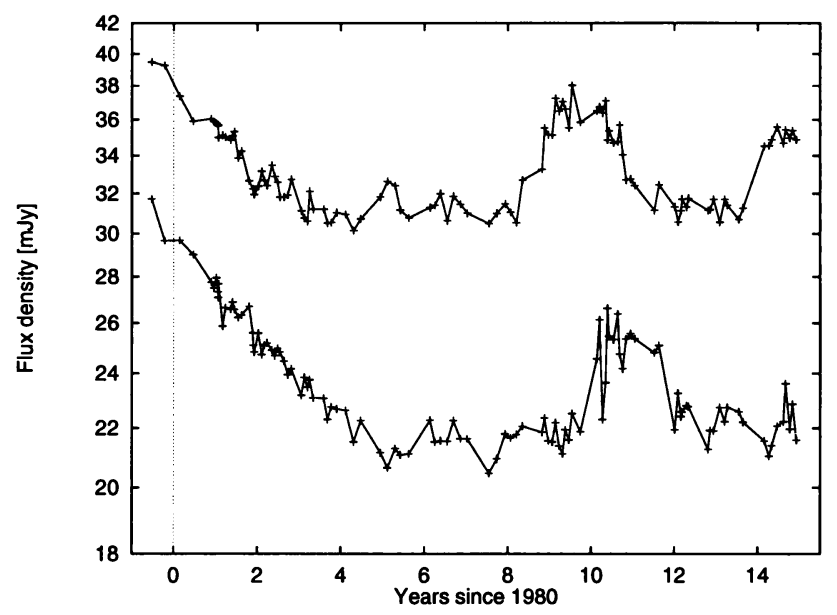

Figure 1: VLA $5 \mathrm{GHz}$ light curves

Figure 2: Chi-squared surface for new light curves: contours start at 280 and increase by 10 in chi-squared

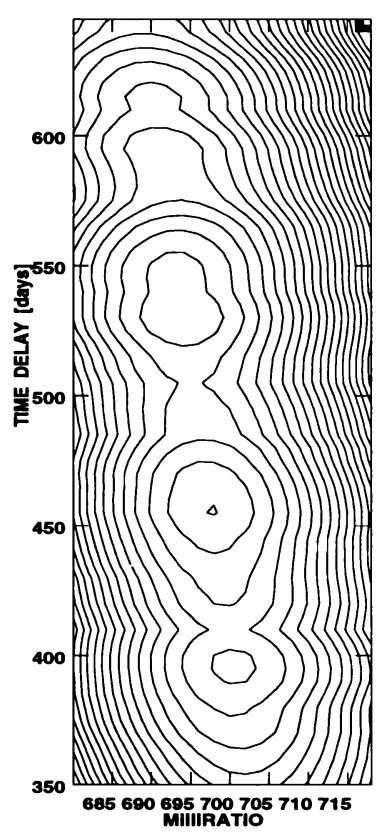

around $\tau=530$ days, as well as around $\tau=400$ days. A Monte Carlo analysis that assumes a gaussian process gives an uncertainty for the new result of \pm 10 days. The Monte Carlo $\chi^{2}$ surfaces do not have the structure of the $\chi^{2}$ surface of Figure 2, so we suspect that this analysis underestimates the uncertainty in the time delay. From inspection of Figure 2, we estimate an uncertainty of \pm 40 days. Rapid variations, especially during the B-image rise in 1990, are responsible for the ambiguity in the delay. Interstellar scintillation may be important, and an analysis that takes this into account is needed.

Pelt et al. (1994) determine the time delay by minimizing the dispersion of the combined light curve. For the 1979-1990 data, the dispersion using only $\mathrm{AB}$ pairs has a minimum at $\tau=533 \pm 40$ days. When the observations on 90Apr10 and 90May07 are removed, the minimum shifts to $\tau=409 \pm$ 23 days. Applying the same analysis to the 1979-1994 data, we find the dispersion minimum is again at 533 days, and it also shifts to 409 days when the $90 \mathrm{Apr} 10$ and $90 \mathrm{May} 07$ observations are removed.

\section{References}

Lehár J., Hewitt J.N, Roberts D.H., \& Burke B.F., 1992, ApJ, 384, 453

Pelt J., Hoff W., Kayser R., Refsdal S., \& Schramm T., 1994, A\&A, 286, 775

Press W.H., Rybicki G.B., Hewitt J.N., 1992a, ApJ, 384, 404

Press W.H., Rybicki G.B., Hewitt J.N., 1992b, ApJ, 384, 416

Walsh D., Carswell R.F., \& Weymann R.J. 1979, Nature, 279, 381 